American Journal of Pharmacology and Toxicology 1 (3): 40-47, 2006

ISSN 1557-4962

(C) 2006 Science Publications

\title{
Role of AP-1 Antagonism in Growth Inhibition of Cervical Cancer Cell Lines by Retinoids
}

\author{
${ }^{1,2}$ Shennan Lu and ${ }^{1,2}$ Doris M. Benbrook \\ ${ }^{1}$ Department of Obstetrics and Gynecology and ${ }^{2}$ Department of Biochemistry and Molecular Biology \\ The University of Oklahoma Health Sciences Center, Oklahoma City, OK 73190
}

\begin{abstract}
Retinoid compounds induce multiple molecular effects that need to be taken into consideration in the development of theses agents as pharmaceuticals for cancer chemoprevention. Inhibition of cancer cell growth by retinoids is thought to occur through at least two mechanisms of transcriptional regulation resulting from direct binding of retinoids to nuclear retinoid receptors (RARs and RXR's). First, the nuclear receptors induce or repress expression of specific genes by direct binding to retinoic acid response elements (RAREs) in gene promoters. Second, the nuclear receptors antagonize transactivation of activator protein-1 (AP-1) promoter elements by the AP-1 transcription factors. The objective of this study was to determine the contributions of RARE transactivation and AP1 antagonism to the mechanism of growth inhibition in cervical cancer cell lines by: the 9-cis isomer of retinoic acid (9-cis-RA), which binds both RARs and RXRs; and a series of synthetic retinoids, called heteraoarotinoids, that possess various receptor specificities and reduced toxicity. The effects of these compounds on reporter gene expression and proliferation were measured in CC-1 and SiHa cell lines stably transfected with RARE or AP-1 reporter plasmids or a retrovirus harboring an inducibile AP-1 protein that is a dominant negative mutant of c-Jun (TAM67). In the CC-1 cell line, which exhibited high AP-1 activity, retinoid growth inhibition significantly correlated with both RARE transactivation and AP-1 repression, was antagonized by superinduction of AP-1 with TPA (12-O-tetradecanoylphorbol-13-acetate) and was enhanced by TAM67. In the SiHa cell line, which exhibited low AP-1 activity, RARE transactivation also significantly correlated with growth inhibition, but AP-1 induction, in contrast to AP-1 repression, correlated with growth inhibition. Furthermore, TPA superinduction of AP-1 did not attenuate retinoid growth inhibition activity in SiHa. In conclusion, repression of AP-1 with retinoids may only be effective against a subset of tumors with high AP-1 activity.
\end{abstract}

Key words: AP-1, retinoids, cervical cancer, growth inhibition

\section{INTRODUCTION}

Retinoic acid (RA) and synthetic analogs, called retinoids, are promising anti-cancer agents based on their abilities to regulate growth, differentiation, apoptosis, angiogenesis and the immune system ${ }^{[1]}$. These activities are mediated by two classes of nuclear retinoid receptors (RARs and RXRs), which act as transcription factors by binding to retinoic acid response elements (RAREs) as dimers ${ }^{[2,3]}$. Natural isomers of RA and synthetic retinoids can selectively bind to and regulate RARE transactivation by retinoid receptors. The all-trans and the 13cis isomers of RA (trans-RA and 13-cis-RA, respectively) bind only to the RAR's while the 9-cis isomer (9-cis-RA) regulates both the RARs and RXRs ${ }^{[4]}$. Since the first isomer studied, trans-RA, was too toxic for most clinical applications, efforts to reduce the toxicity by designing synthetic retinoids selective for receptors and activities were pursued by restricting the flexibility of the tetraene side chain of trans-RA through incorporation into aromatic rings ${ }^{[5,6]}$. Thousands of different aromatic retinoids, called arotinoids, have been synthesized and many were found to be selective for retinoid receptors and retinoid activities ${ }^{[7,8]}$. The first arotinoid thoroughly evaluated, TTNPB, was found to be 1000fold more toxic than trans-RA ${ }^{[9-11]}$. Incorporation of a heteroatom into the cyclic ring of arotinoids resulting in compounds called heteroarotinoids reduced the toxicity 1000fold in the diaryl TTNPB structure and 3000 fold in the monoaryl heteroarotinoid structure ${ }^{[11]}$. Therefore, this class of compounds has the potential for clinical application as anticancer drugs. In support of this, the Chemoprevention Working Group to the American Association for Cancer Research concluded that retinoids are considered one of the most promising classes of cancer chemoprevention agents ${ }^{[12]}$.

Although the toxicities have been reduced, the complexities of retinoid activities still need to be deciphered in order to understand and optimize the activities involved in the anti-cancer mechanisms. In addition to RARE transactivation, retinoids inhibit transactivation by the Activator Protein 1 (AP1) transcription factors which include the Jun, Fos and CREB families of transcription factors, as well as other leucine zipper proteins ${ }^{[13]}$. AP-1 transcription factors also bind to DNA response elements (AP-1 sites) as dimers and regulate growth, differentiation and apoptosis ${ }^{[14]}$. Induction of AP-1 is an immediate early response in cells stimulated to grow or stimulated with a stress-induced signal, such as DNA damage ${ }^{[14]}$. The AP-1 transcription factor called cJun is considered an oncogene and is highly regulated through posttranslational modifications including phosphorylation and oxidation of the transcriptional activation and DNA binding

Corresponding Author: Dr. Doris M. Benbrook, University of Oklahoma Health Sciences Center, 975 NE $10^{\text {th }}$ Street; Room 1372. Oklahoma City, OK 73104, Tel.: 405-271-5523; Fax: 405- 271-3874 
domains $^{[13]}$. Activation of protein kinase C (PKC) with the tumor promoter, 12-O-tetradecanoylphorbol-13-acetate (TPA) induces cJun activity by causing dephosphorylation of sites that negatively regulate cJun DNA binding activity ${ }^{[15]}$. On the other hand, a mutant of cJun (TAM67) that is deleted in the transactivation domain, but retains the dimerization and DNA binding domains, can quench cJun and AP-1 activity ${ }^{[16]}$.

The involvement of AP-1 in growth stimulation, suggests that retinoid repression of AP-1 function is involved in mechanism of retinoid growth inhibition. In support of this, retinoids that lack RARE transactivation, but that are still capable of AP-1 repression, were found to be equally effective as RA in inhibiting the growth of 3 breast cancer cell lines ${ }^{[17]}$. Also, overexpression of cJun was found to induce retinoid resistance the MCF7 breast cancer cell line ${ }^{[18]}$. A separate study, however demonstrated that the KNCR neuroblastoma cell line was not inhibited by AP-1 selective retinoids, despite sensitivity to RARE transactivating retinoids ${ }^{[19]}$. Therefore, it is likely that other retinoid activities contribute to the mechanism of growth inhibition. In support of this, our previous studies demonstrated that retinoid RARE transactivation significantly correlated with growth inhibition in the CC-1 cervical cancer cell line ${ }^{[11,20]}$. AP1 repression therefore, may not be the only mechanism of retinoid growth inhibition and designing retinoids specifically targeted at AP-1 repression may be less efficient than designing retinoids targeted to a larger subset of activities involved in the mechanism of retinoid growth inhibition. The objective of this study was to determine if retinoids could inhibit cervical cancer cell growth through AP-1-independent mechanisms.

\section{MATERIALS AND METHODS}

Cell lines and drug treatment: $\mathrm{CC}-1$ and $\mathrm{SiHa}$ cell cultures were maintained in Eagle's minimal essential media (MEM) containing Earle's salts and L-glutamine (Cellgro, Mediatech, Herndon, VA) supplemented with nonessential amino acids, sodium pyruvate, anti-bioticanti-mycotic and 10\% fetal bovine serum. Phoenix A cell cultures were maintained in Dubeccal's modified eagle media (DMEM) containing pyridoxine hydrochloride and high glucose supplemented with L-glutamine, anti-biotic-antimycotic and 10\% fetal bovine serum. Media were replenished every 2 days. The synthetic retinoids (heteroarotinoids - gifts of K. Darrell Berlin, Oklahoma State University) were synthesized as previously described ${ }^{[11,21,22]}$. The 9-cis-RA (BIOMOL, Plymouth Meeting, PA), heteroarotinoids and TPA (Sigma, St. Louis, MO) were dissolved in dimethyl sulfoxide (DMSO) as $1000 \times$ stock solutions, so that the final concentration of DMSO in all cultures was less than $0.1 \%$, which is not cytotoxic and does not induce differentiation. Drugs were replenished every 2 days, with the media. The reporter cell lines were established by co-transfection of the pColCAT reporter plasmid, which contains the collagenase AP-1 DNA binding site and the thymidine kinase (tk) promoter driving the chloramphenicol acetyl transferase (CAT) gene, or the RARE-tk-CAT reporter plasmid, which contains the RARE from the RAR $\beta$ gene in place of the AP- 1 site in the same plasmid. CC-
1 and $\mathrm{SiHa}$ cultures were plated in 6-well plates at a concentration of $5 \times 10^{5}$ well $^{-1}$ before the day of transfection. The next day, $2 \mu \mathrm{g}$ of each reporter

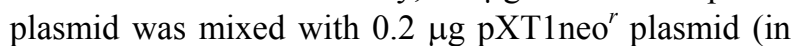
order to convey the Geneticin resistance) and diluted in $100 \mu \mathrm{l}$ serum-free MEM media containing $3 \mu \mathrm{l}$ of Fugene 6 (Roche Molecular Biochemicals, Indianapolis, IN). Geneticin-resistant colonies were isolated and tested for CAT expression.

RARE and AP-1 transactivation and repression: The reporter cell lines were plated in 6 -well plates at $5 \times 10^{5}$ cells well $^{-1}$ and incubated overnight to allow cells to become attached. The next day, the media were replenished and drugs were added. After $48 \mathrm{hrs}$ of treatment, cell lysates were prepared according to published procedures ${ }^{[23]}$ and assayed for the CAT expression using the CAT ELISA Kit (Roche Molecular Biochemicals, Indianapolis, IN). The protein concentration of each lysate was determined with the Protein Assay reagent (BioRad, Richmond, CA). All results were normalized for protein concentration.

Growth assays: Cultures were plated in 96-well microtiter plate at 400 cells well $^{-1}$ and incubated over night. The next day, the media in each well were carefully removed with a multichannel pipetman and replaced with media containing appropriate concentration of drugs. For the 3-day growth assay, the media and drug were not replenished for the duration of the treatment period. For the 7-day growth assay, the media and drugs were replenished every two days. A the end of the treatment period, growth was assayed by the MTT (3-(4,5dimethylthiazol- 2-yl)- 5- (3- carboxymethoxyphenyl)- 2Htetrazolium inner salt) assay (Promega, Madison, WI). All treatments were performed in triplicate. The growth index was determined by dividing the average OD of the treated wells by the average OD of the control untreated wells. The results were presented as the average and standard deviation of 3 to 10 experiments.

Retroviral expressions of dominant negative cJun: Eight micrograms of the TAM67 expression vector (gift from Dr. Michael Birrer, National Institutes of Health) was transfected into Phoenix A cells using Fugene 6. After $48 \mathrm{hrs}$, the media was replenished and cells were incubated at $32^{\circ} \mathrm{C}$ for $48 \mathrm{hrs}$ to accumulate virus particles. The media was then collected, spun at 10,000 rpm for $10 \mathrm{~min}$ to eliminate any phoenix A cells and then either directly used to infect other cells or frozen at $-70{ }^{\circ} \mathrm{C}$ for future use. Before infection, $\mathrm{CC}-1$ and $\mathrm{SiHa}$ cells were plated at $5 \times 10^{5}$ cells well ${ }^{-1}$ on 6 -well plates, $24 \mathrm{hrs}$ before infection. The media in each well were then removed and a cocktail consisting of $0.5 \mathrm{~mL}$ virus-containing media and $6 \mu \mathrm{g}$ $\mathrm{mL}^{-1}$ polybrene (hexadimethrine bromide) (Sigma, St. Louis, MO) in MEM media. After $6 \mathrm{hrs}$ of incubation at $37^{\circ} \mathrm{C}$, the cocktail was removed and replaced with fresh MEM media. The cultures were then incubated at $37^{\circ} \mathrm{C}$ for another $48 \mathrm{hrs}$ to allow the expression of blasticidin $\mathrm{S}$ deaminase. The cells in each well were then trypsinized and plated at low density and 
blasticidin $\mathrm{S}$ was added to isolate resistant colonies. To induce TAM67 expression, $1 \mu \mathrm{g} \mathrm{mL}^{-1}$ of doxycycline was added to the culture media simultaneously with retinoid or TPA treatment.

Electrophoretic mobility shift assay (EMSA): A double stranded oligocleotide probe encoding the collagenase gene promoter AP-1 response element (GATCCTAAAGCATGAGTCAGACACCG) was radioactively labeled using MuMLV Reverse Transcriptase and $\alpha-\mathrm{P}^{32}$-dCTP (New England Nuclear, City, State) and used in the binding assay. A probe mixture was prepared by mixing 2 $\mu \mathrm{l}$ of the labeled oligonucleotide, $88 \mu \mathrm{l}$ of the Gel-Retardation Buffer (25 mM HEPES pH 7.9, 150 mM KCl, 1 mM EDTA, 5 $\mathrm{mM}$ DTT and 10X Denhardts reagent) and $10 \mu \mathrm{l} 2.5 \mathrm{mg} \mathrm{mL}^{-1}$ poly-dIdC. For each binding reaction, exactly $1 \mu \mathrm{g}$ of nuclear proteins was mixed with $15 \mu \mathrm{l}$ of GRB, $5 \mu \mathrm{l}$ probe mixture and appropriate amount of nuclear extraction buffer so that the final volume was $25 \mu$. The mixture was incubated at $4^{\circ}$. C for 20 minutes. Reactions containing 100 fold excess specific competitor (unlabeled same oligonucleotide) and non-specific (unlabeled and mutated AP-1 binding site) and antibody against cJun were also prepared to test the specificity of binding. All reactions were then directly loaded onto an $8 \%$ non-denaturing polyacrylamide gel and electrophoresed for $2 \mathrm{hrs}$ at 200 volt in 0.5 XTBE (Tris, Borate and EDTA) buffer. The gel was dried and the bands were detected and quantitated by either using a phosphoimage system (Dynamic Molecules) or exposing a Kodak X-ray film and densitometric scanning of the bands. Each experiment was repeated between 1 to 3 times to confirm the results.

Western analysis: Cultures in 6-well plates were trypsinized and collected into $1.5 \mathrm{~mL}$ eppendorf tubes and washed twice with PBS. The pellets were resuspended in suspension buffer $\left(0.1 \mathrm{~mol} \mathrm{~L}^{-1} \mathrm{NaCl}, 10\right.$ mmol L ${ }^{-1}$ Tris-HCl (pH7.6), 1 mmol L ${ }^{-1}$ EDTA (pH8.0), $1 \mu \mathrm{g} \mathrm{mL}^{-1}$ Aprotinin, $100 \mu \mathrm{g} \mathrm{mL}^{-1}$ PMSF) and lysed by adding SDS loading buffer $\left(0.1 \mathrm{~mol} \mathrm{~L}^{-1}\right.$ Tris$\mathrm{HCl}$ (pH6.8), $0.2 \mathrm{~mol} \mathrm{~L}^{-1}$ DTT, 4\% SDS, 20\% Glycerol, $0.2 \%$ Bromophenol Blue). Samples were then heated at $100^{\circ} \mathrm{C}$ for $10 \mathrm{~min}$, vortexed vigorously and frozen at $20^{\circ} \mathrm{C}$. Samples were quickly thawed and centrifuged at $12,000 \mathrm{rpm}$ for $15 \mathrm{~min}$ before loaded on $10 \%$ denaturing gel $\left(0.375 \mathrm{~mol} \mathrm{~L}^{-1}\right.$ Tris- $\mathrm{HCl}(\mathrm{pH} 8.8), 0.1 \%$ SDS) in Tris-Glycine buffer ( $3.03 \mathrm{~g} \mathrm{~L}^{-1}$ Tris, $14.4 \mathrm{~g} \mathrm{~L}^{-1}$ Glycine, 0.1\% SDS, pH 6.6) and electrophoresed for 1 hour. Separated proteins were transferred from gel to PVDF membrane (Millipore IMMOBIOLONTM-P) using a BioRad TRANSBLOT semi-dry transfer cell at $200 \mathrm{~mA}$ for $30 \mathrm{~min}$. The membrane was then blocked in $10 \%$ blocking solution $(10 \%$ dry nonfat milk, $20 \mathrm{mmol}$ $\mathrm{L}^{-1}$ Tris-HCl (pH 8.0), $100 \mathrm{mmol} \mathrm{L}^{-1} \mathrm{NaCl}, 0.05 \%$ Tween-20) for 1 hour at room temperature, washed with washing buffer $\left(20 \mathrm{mmol} \mathrm{L}^{-1}\right.$ Tris- $\mathrm{HCl}$ (pH8.0), $100 \mathrm{mmol} \mathrm{L}^{-1} \mathrm{NaCl}, 0.05 \%$ Tween-20, $2 \mathrm{mmol} \mathrm{L}^{-1}$ $\left.\mathrm{CaCl}_{2}\right)$ for $15 \mathrm{~min}$, incubated in primary antibody $(1 \mathrm{mg}$
$\mathrm{mL}^{-1}$ in washing buffer) for 1 hour, washed again in washing buffer for $15 \mathrm{~min}$, incubated in secondary antibody (Anti-Rabbit-POD from Roche Molecular Biochemicals, Indianapolis, IN, $0.02 \mathrm{U} \mathrm{mL}^{-1}$ in washing buffer) for 1 hour and finally washed 3 times in washing buffer. The membrane was soaked in substrate solution (Amersham Pharmacia Biotech, Piscataway, $\mathrm{NJ})$ for 1 minute before film exposure. Each Western was repeated once to confirm the results.

Statistical analysis: Statistical analysis was performed using GraphPad Software (Prism).

\section{RESULTS}

Correlation of RARE and AP-1 transactivation with growth inhibition: Transactivation of RARE and AP-1 DNA elements by transcription factors endogenous to cancer cells was measured in CC-1 and SiHa cell lines that were stably transfected with reporter plasmids. These plasmids encode the chloramphenicol acetyl transferase (CAT) gene under the control of an RARE or an AP-1 DNA binding sites, so that the levels of CAT produced in the cells represent a measure of transcriptional regulation from the RARE or AP-1 DNA binding sites. The CC-1 and SiHa RARE reporter cell lines exhibited similar dose-response curves of RARE transactivation by 9-cis-RA that were saturated at the 6-7 fold induction level in the $\mu \mathrm{M}$ concentration range (Fig. 1A). In contrast, AP-1 induction by TPA was 10 fold higher in the CC1 reporter cell line than in the SiHa reporter cell line, but both reached saturation between 0.4 and $0.8 \mathrm{ng} \mathrm{mL}^{-1} \mathrm{TPA}$ (Fig. 1B). This 10-fold difference was consistently observed with several CC-1 and SiHa AP-1 reporter cell lines tested. These reporter cell lines were used to screen a series of 9 synthetic retinoids and the natural 9-cis-RA for their ability to regulate RARE and AP-1 transactivation and to inhibit growth in the same cell lines. Linear regression analysis demonstrated a statistically significant direct correlation between reductions in the growth indexes and RARE transactivation caused by the retinoids in both $\mathrm{CC}-1$ ( $(\mathrm{P}=0.0003)$ and $\mathrm{SiHa}(\mathrm{P}=0.0026)$ (Fig. $1 \mathrm{C}$ and $1 \mathrm{D}$, respectively). Although retinoid repression of AP-1 induction directly correlated with growth inhibition in CC-1 (P $=0.0003$ ), it inversely correlated with growth inhibition in $\mathrm{SiHa}$ $(\mathrm{P}=0.145)$ (Fig. 1E and 1F, respectively).

Mutual antagonism of retinoids and TPA on RARE and AP-1 transactivation: The natural 9-cis-RA exhibited a dose responsive repression of AP-1 activity in the CC-1 and $\mathrm{SiHa}$ AP-1 reporter cell lines (Fig. 2A and 2B, respectively). TPA concentrations that induced maximal AP-1 activity reduced, but did not prevent dose-responsive AP-1 antagonism caused by 9cis-RA in both cell lines. Conversely, 9-cis-RA concentrations that maximized RARE transactivation reduced, did not prevent TPA-induction of AP-1 activity in the CC-1 reporter cell line (Fig. 1B).

The DNA binding activities of the AP-1 transcription factors in CC-1 and SiHa, were evaluated with EMSA's performed on nuclear protein extracts isolated from cultures 
A

RARE

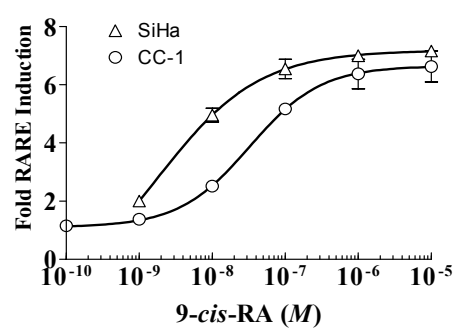

B

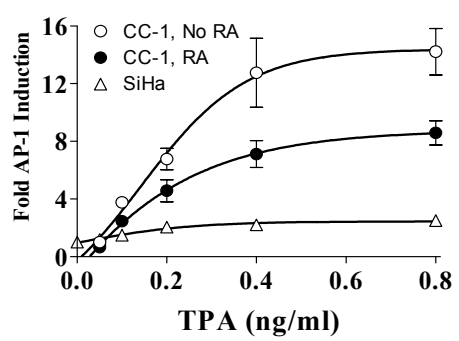

C

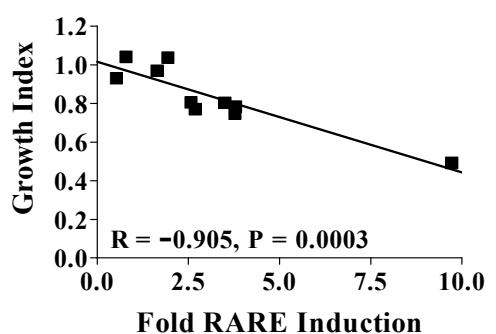

D

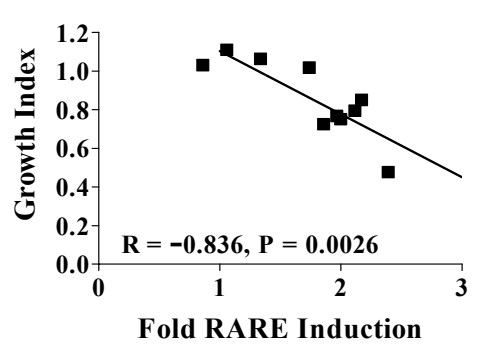

$\mathbf{E}$

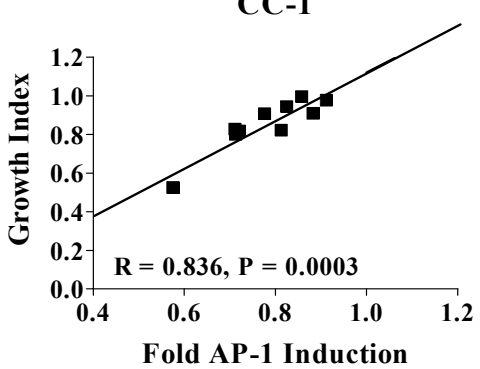

F

SiHa

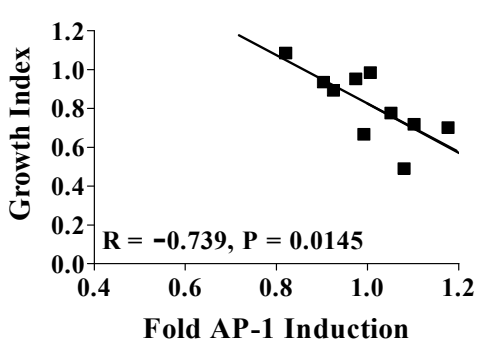

Fig. 1: Correlation of retinoid regulation of RARE transactivation, AP-1 transactivation and growth. A, RARE transactivation by 9-cis-RA in CC-1 and SiHa RARE reporter cell lines. B, AP-1 transactivation by TPA in CC-1 and SiHa AP-1 reporter cell lines. The effects of 10 $\mu \mathrm{M}$ 9-cis-RA on TPA transactivation of AP-1 is shown for CC-1. C - F, Correlation analysis, The reporter cell lines CC-1 RARE (C), the SiHa RARE (D), CC-1 AP-1 (E) and SiHa AP-1 (D) were treated with 10 different retinoids. The growth index after 3 days of treatment and the RARE transactivation after 2 days of treatment were plotted and correlated using GraphPad Software

A

C C-1

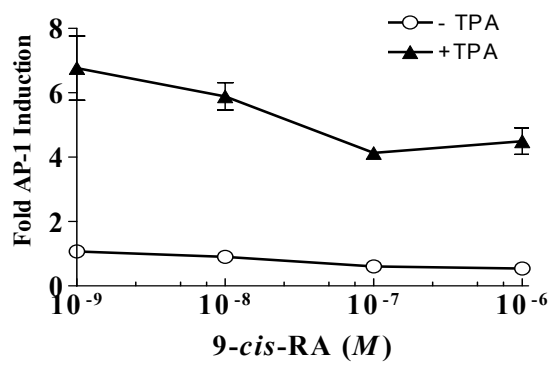

B

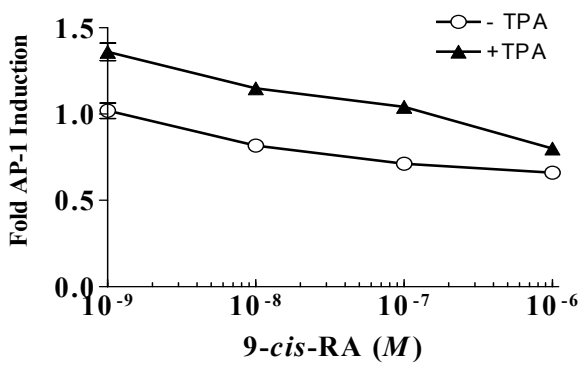

C $\quad \begin{array}{llllllllllll} & 0 & 1 & 2 & 3 & 4 & 5 & 6 & 7 & 8 & 9 & 10\end{array}$

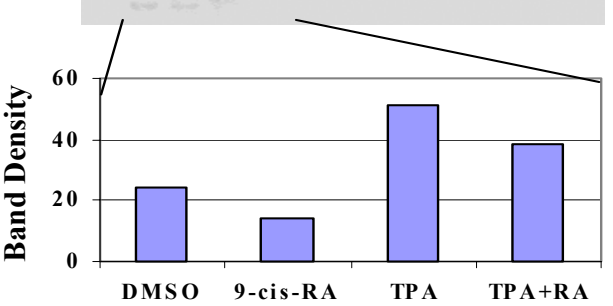

D $\quad \begin{array}{llllllllllll}0 & 1 & 2 & 3 & 4 & 5 & 6 & 7 & 8 & 9 & 10\end{array}$

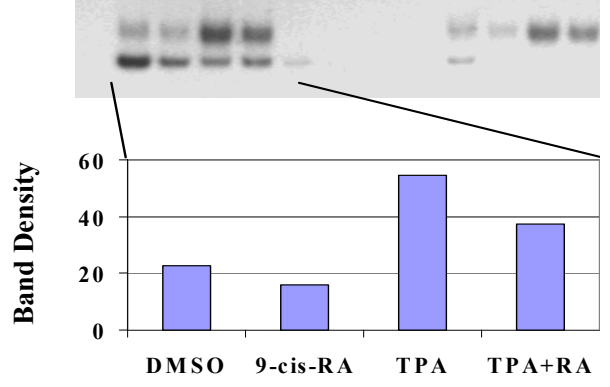

Fig. 2: Mutual antagonism of 9-cis-RA and TPA on AP-1 Transactivation and DNA binding. A-B, Repression of AP-1 induction by 9-cis-RA in the absence and presence of $0.4 \mathrm{ng} \mathrm{mL}{ }^{-1}$ TPA in the CC-1 AP-1 (A) and SiHa AP-1 (B) reporter cell lines. C-D, EMSAs of the collagenase AP-1 DNA binding site oligonucleotide incubated alone (lane 0 ), or with nuclear extracts from cultures that were untreated (lanes $1,5,9)$, treated with $10 \mu \mathrm{M}$ 9-cis-RA (lanes 2, 6, 10), with $0.4 \mathrm{ng} \mathrm{mL}^{-1} \mathrm{TPA}$ (lanes 3, 7 and 11), or both agents (lanes 4, 8, 12) for 2 days. Bar graphs below the EMSAs represent denistometric quantitation of the specific bands in lanes 1 (DMSO), lane 2 (9-cis-RA), lane 3 TPA) and lane 4 (TPA + RA) 

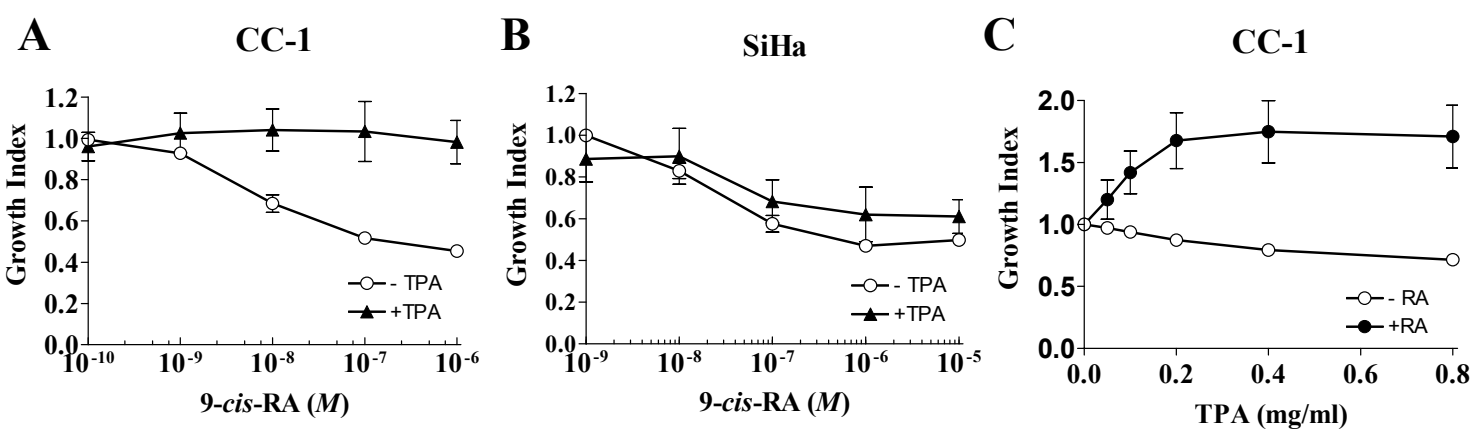

D

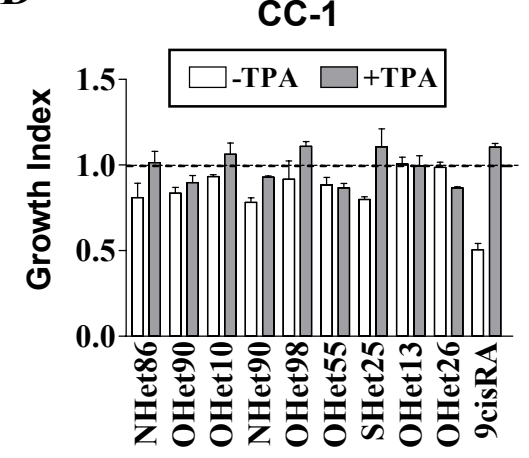

$\mathbf{E}$

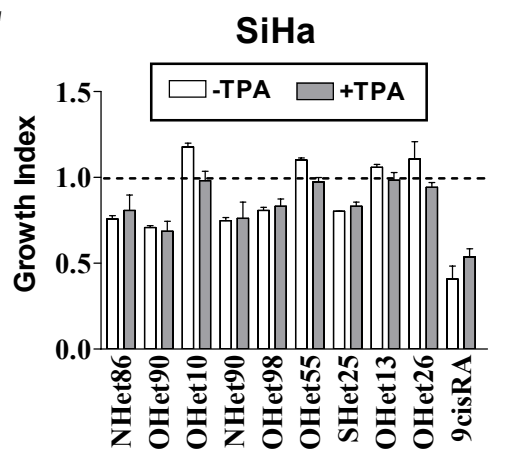

Fig. 3: TPA antagonizes retinoid growth inhibition in CC-1, but not in SiH.Seven day assays of 9-cis-RA growth inhibition in the absence and presence of $0.4 \mathrm{ng} \mathrm{mL} \mathrm{m}^{-1} \mathrm{TPA}$ in CC-1 (A), SiHa (B). TPA growth inhibition in the absence and presence of 9-cis-RA in CC-1 (C). TPA effects on growth inhibition by a variety of retinoids listed on the x-axis labels in CC-1 (D) and SiHa (E)

treated with 9-cis-RA and/or TPA (Fig. 2C and 2D). In comparison to the untreated control (lane 1), 9-cis-RA treatment decreased (lane 2) and TPA treatment increased (lane 3), AP-1 DNA binding. Simultaneous treatment with both agents resulted in intermediate levels of DNA binding (lane 4), demonstrating mutual antagonism of RA and TPA on AP-1 DNA binding activity. The intensities of the specific bands in lanes 1 through 4 were quantitated with densitometric scanning (Fig. 2C and 2D, bar graphs below each EMSA gels), which confirmed this visual interpretation. The specificity of the retarded bands was confirmed by competition with a 100 -fold excess of non-radioactively-labeled oligonucleotide containing the AP-1 DNA binding site (Fig. 2C and 2D, lanes 5-8) and lack of competition by an oligonucleotide containing an unrelated DNA binding site sequence (Fig. 2C and 2D, lanes 9-12).

Retinoid Growth inhibition in the context of TPAantagonized AP-1 repression: The mutual antagonism of TPA and retinoids on AP-1 activity was utilized to test if retinoids can inhibit growth in the absence of AP-1 repression. Cultures were treated with a range of 9-cis-RA concentrations in the presence and absence of the concentration of TPA that saturated AP-1 induction $\left(0.4 \mathrm{ng} \mathrm{mL} \mathrm{mL}^{-1}\right)$. TPA alone inhibited the growth of $\mathrm{CC}-1$ by $8 \%$ and $\mathrm{SiHa}$ by $15 \%$. After normalizing for the effects of TPA alone, it was still apparent that TPA antagonized retinoid growth inhibition in CC-1 (Fig. 3A), but had only slight effect in SiHa (Fig. 3B). Since TPA activation of PKC has many other effects besides the activation of cJun and AP-1 transactivation, the TPA interactive effects with retinoids may be due to AP-1 independent effects of TPA. To determine if TPA and 9-cis-RA effects were additive, indicating that they work through the same mechanism of action, or if the were synergistic, indicating that they work through complementary mechanisms of action, the fractional product method was used ${ }^{[24]}$. In this method if the effect of combined treatment $\left(\mathrm{E}_{9-\text {-is-RA+TPA }}\right)$ is greater than the product of the effects of the individual treatments ( $\left.\mathrm{E}_{9-c i s-\mathrm{RA}} \mathrm{X} \mathrm{E}_{\mathrm{TPA}}\right)$, the two drugs are considered to work synergistically. In CC-1, the products of growth indexes of TPA alone and 9-cis-RA alone were not significantly different from the growth indexes of the combined treatment at all 9-cis-RA doses tested. Therefore the antagonism of 9-cis-RA growth inhibition in CC-1 is additive and not synergistic, suggesting that both agents regulate $\mathrm{CC}-1$ growth through the same molecular mechanism of actions, i.e. regulation of AP-1 activity.

The ability of saturating levels of TPA to counteract growth repression by the 9 synthetic retinoids was evaluated in the $\mathrm{CC}-1$ and $\mathrm{SiHa}$ reporter cell lines and the effects of the combined treatments were normalized for the effects of TPA alone. When used in combination, TPA alleviated growth repression by most compounds in CC-1 (Fig. 3D), but not in SiHa (Fig. 3E).

Retinoid growth inhibition in the context of quenched AP1: To further test the hypothesis that retinoids can regulate growth independent of their AP-1 repression, a dominant negative mutant of one of the AP-1 transcription factors, called cJun, was utilized to quench AP-1 activity. This mutant, called TAM67, is deleted in the protein domain responsible for 

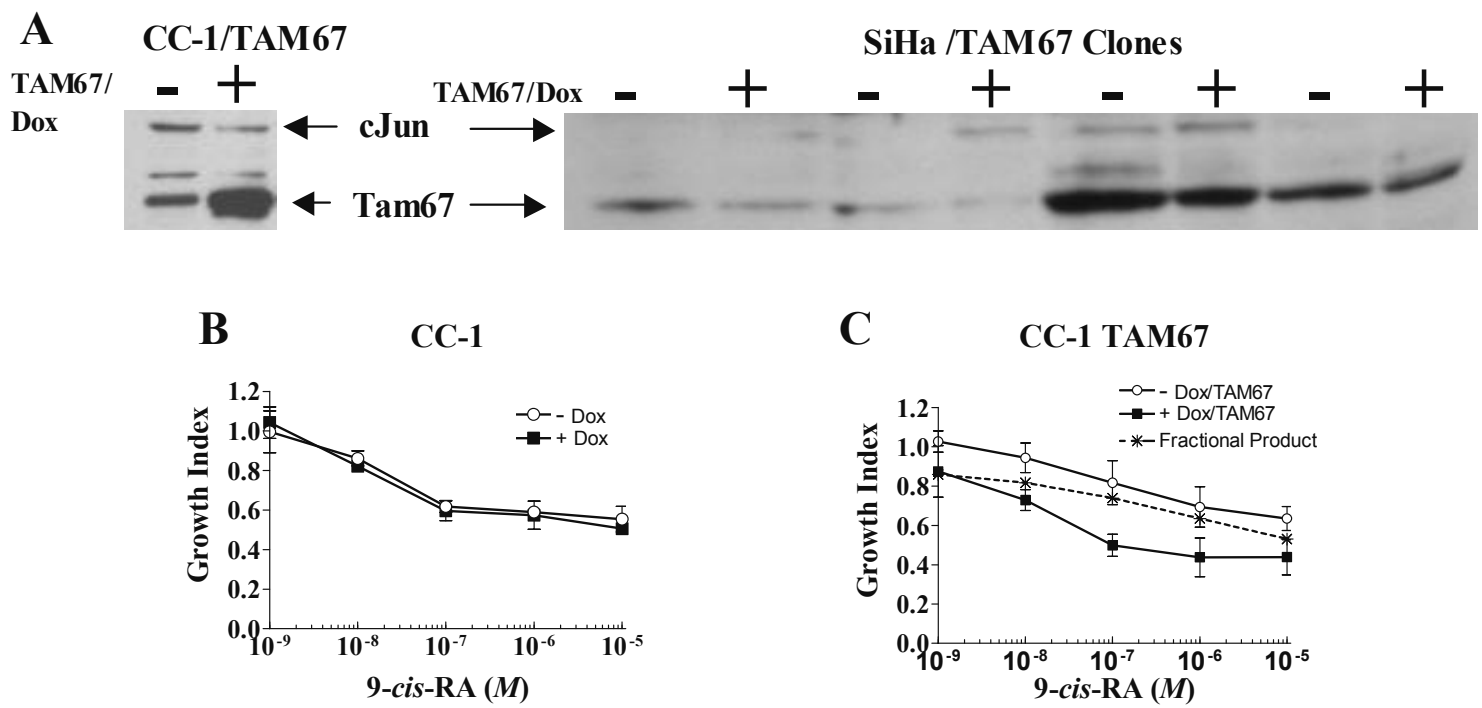

Fig. 4: Dominant negative cJun enhances retinoid growth inhibition in 2 out of 3 cell lines. A, Western Analysis of TAM67 and cJun expression in the absence (-) and presence $(+)$ of doxycycline (dox). B, 9-cis-RA growth inhibition in the absence and presence of dox in CC-1 cultures harboring empty retrovirus (B) or subclones harboring TAM67 retrovirus (C). The dashed line with astericks represents the fractional product calculated by multiplying the effects of dox alone by the effects of 9-cis-RA alone

transactivation and therefore will dimerize with the full-length cJun and all of the other AP-1 transcription factors, resulting in nonproductive complexes that can bind DNA but cannot transactivate AP-1 sites ${ }^{[16]}$. The TAM67 mutant was introduced into $\mathrm{CC}-1$ and $\mathrm{SiHa}$ cell lines using a retrovirus vector that contains the Blasticidine $\mathrm{S}$ resistance gene and Blasticidine $\mathrm{S}$ resistant colonies were isolated. The retrovirus was induced to express TAM67 using a drug called doxycycline (dox). In CC1 cultures containing the TAM67 retrovirus, Western Analysis demonstrated leaky expression of TAM67 in the absence of dox treatment and induction of TAM67 in the presence of dox (Fig. 4A). Consistent with the known positive autoregulation of cJun expression, dox decreased the expression of cJun demonstrating loss of cJun transactivation activity in the presence of TAM67 (Fig. 4A). Despite leaky expression of TAM67 in SiHa cultures containing the retrovirus (SiHaT67), the TAM67 protein was not inducible by dox treatment in 8 separate clones isolated and evaluated (4 of the clones are shown in Fig. 4A). The SiHa clones exhibited a very low to undetectable levels of wild type cJun expression in comparison to $\mathrm{CC}-1$.

The effects of TAM67 expression on 9-cis-RA growth inhibition was tested by inducing TAM67 expression with dox treatment. Atlhough dox had no effect on growth of the parental CC-1 cell line (Fig. 4B), it significantly enhanced the growth inhibition caused 9-cis-RA in the CC1 TAM67 clone (Fig. 4C). This effect was due to more than an additive effect of dox and 9-cis-RA on CC1 TAM67 growth because the growth indexes in the combined treatment curve were normalized for the effect of dox alone. To further demonstrate that the effects of doxinduced TAM67 expression and 9-cis-RA treatment were synergistic and not additive, the fractional product method was used $^{[24]}$. The product of the growth indexes of dox and 9-cisRA single agents was plotted on the same graph as the growth index curves of 9-cis-RA alone and in combination with dox (but not normalized for dox alone) (Fig. 4E). The combined treatment curve is clearly below the calculated fractional product curve (dashed line), demonstrating synergistic effects of TAM67 and 9-cis-RA and indicating that these two agents act through separate but complementary mechanisms of action. As expected, TAM67 expression and dox treatment had no effects in $\mathrm{SiHa}$ (data not shown), most likely due to its low level of endogenous AP-1 activity.

\section{DISCUSSION}

The results of this study demonstrate that the contribution of AP-1 antagonism to the mechanism of retinoid growth inhibition is dependent upon the cellular environment. While RARE transactivation correlated with growth inhibition in both cell lines, AP1 antagonism only appeared to play a major role in the context of high AP-1 activity. In the CC-1 cell line, which exhibited high levels of AP-1 activity, the contribution of AP-1 antagonism to retinoid growth inhibition was demonstrated by the findings that: 1) AP-1 antagonism significantly correlated with growth inhibition activities of 10 retinoids, 2) TPA induction of AP-1 additively antagonized retinoid growth inhibition, and 3) TAM67 inhibition of AP-1 enhanced retinoid growth inhibition. The SiHa cell line, which exhibited a low level of AP-1 however, did not exhibit significant contribution of AP-1 antagonism to the mechanism of retinoid growth inhibition. In $\mathrm{SiHa}, \mathrm{AP}-1$ antagonism inversely correlated with growth inhibition and TPA induction of AP-1 did not significantly attenuate retinoid growth inhibition. Since RARE transactivation correlated with growth inhibition in both $\mathrm{CC}-1$ and 
$\mathrm{SiHa}$, it is likely that this activity is primarily responsible for growth inhibition in $\mathrm{SiHa}$ and is overshadowed by AP-1 antagonism in CC-1.

The SiHa cell line exhibited the greatest sensitivity to retinoid growth inhibition, but also exhibited the lowest level of basal and induced AP-1 activity. TPA-induction in SiHa was 10-fold lower than in CC-1 and Western Analysis confirmed a very low level of cJun expression in $\mathrm{SiHa}$ in comparison to CC1. This trend of lower AP-1 associated with increased retinoid sensitivity was also observed in the retinoid-sensitive MCF7 breast carcinoma cell line, which has low basal AP-1 activity, but that can be made retinoid-resistant by overexpression of cJun $^{[18]}$. 9-cis-RA dose-responsively repressed the low level of AP-1 activity in SiHa, this activity inversely correlated with growth inhibition. This might be explained by the ability of AP1 to induce apoptosis in specific cellular environment ${ }^{[13,25]}$.

\section{CONCLUSION}

The transcriptional mechanism of retinoid growth inhibition is dependent upon the cellular context. In a cellular environment with high AP-1 activity, AP-1 antagonism can play a major role in retinoid growth inhibition, but in a cellular environment with low AP-1 activity, AP-1 antagonism can counteract retinoid growth inhibition. Therefore, development of retinoids to specifically target AP-1 may only be effective against a subset of tumors with high AP-1 activity.

\section{ACKNOWLEDGEMENTS}

This work was supported by a grant from the National Institutes of Health RO1 CA 77711 and a Postdoctoral Fellowship from the Cancer Research Foundation of America (CRFA).

\section{REFERENCES}

1. Smith, M.A., D.R. Parkinson, B.D. Cheson and M.A. Friedman, 1992. Retinoids in Cancer Therapy. J. Clin. Oncol., 10: 839-864.

2. Guruswamy, S., S. Lightfoot, M. Gold, R. Hassan, K.D. Berlin, R.T. Ivey and D.M. Benbrook, 2001. Effects of retinoids on cancerous phenotype and apoptosis in organotypic culture of ovarian carcinoma. J. Nat. Cancer Inst., 93: 516-525.

3. Petkovich, M., 1992. Regulation of gene expression by vitamin A: The role of nuclear retinoic acid receptors. Annu. Rev. Nutr., 12: 433-471.

4. Schrader, M., A. Wyss, L.J. Sturzenbecker, J.F. Grippo, P. LeMotte and C. Carlberg, 1993. RXR-dependent and RXR-independent transactivation by retinoic acid receptors. Nucl. Acids. Res., 21: 1231-1237.

5. Silverman, A.K., C.N. Ellis and J.J. Voorhees, 1987. Hypervitaminosis A syndrome: A paradigm of retinoid side effects. J. Am. Acad. Dermatol., 16: 1027-1039.
6. Gale, J.B., 1993. Recent Advances in the Chemistry and Biology of Retinoids. In Luscombe, G.P.E.a.D.K. (Ed.), Progress in Medicinal Chemistry. Elsevier Science B.V., Amsterdam, 30: 1-55.

7. Nagpal, S. and R.A.S. Chandraratna, 2000. Recent developments in receptor-selective retinoids. Curr. Pharm. Des., 6: 919-931.

8. Benbrook, D.M., S. Subramanian, J.B. Gale, S. Liu, C.W. Brown, M.F. Boehm and K.D. Berlin, 1998. Synthesis and characterization of heteroarotinoids demonstrate structure specificity relationships. J. Med. Chem., 41: 3753-3757.

9. Lindamood, C., III, F.O. Cope, D.L. Dillehay, M.P. Everson, H.D. Giles, E.W. Lamon, D.J. McCarthy, J.L. Sartin and D.L. Hill, 1990. Pharmacological and toxicological properties of arotinoids SMR-2 and SMR-6 in mice. Fund. Appl. Toxic., 14: 15-29.

10. Lindamood, C.I., D.L. Dillehay, E.W. Lamon, H.D. Giles, Y.F. Shealy, B.P. Sani and D.L. Hill, 1988 Toxicologic and Immunologic evaluations of N-(All-transRetinoyl)_dL-leucine and N-(all-trans-Retinoyl)glycine. Toxicol. Appl. Pharamcol., 96: 279-295.

11. Benbrook, D.M., M.M. Madler, L.W. Spruce, P.J. Birckbichler, E.C. Nelson, S. Subramanian, G.M. Weerasekare, J.B. Gale, M.K.Jr. Patterson, B. Wang, W. Wang, S. Lu, T.C. Rowland, P. DiSilvestro, C.III Lindamood, D.L. Hill and K.D. Berlin, 1997. Biologically active heteroarotinoids exhibit anticancer activity and decreased toxicity. J. Med. Chem., 40: 3567-3583.

12. Alberts, D.S., O.M. Colvin, A.H. Conney, V.L. Ernster, J.E. Garber, P. Greenwald, L. Gudas, K.W. Hong, G.J. Kelloff, R.A. Kramer, C.E. Lerman, D.J. Mangelsdorf, A. Matter, J.D. Minna, W.G. Nelson, J.M. Pezzuto, F. Prendergast, V.W. Rusch, M.B. Sporn, L.W. Wattenberg and I.B. Weinstein, 1999. Prevention of cancer in the next millennium: Report of the chemoprevention working group to the American Association for Cancer Research. Cancer Res., 59: 4743-4748.

13. Karin, M., Z. Liu and E. Zandi, 1997. AP-1 function and regulation. Cur. Op. Cell Biol., 9: 240-246.

14. Angel, P. and M. Karin, M. 1991. The role of jun, fos and the AP-1 complex in cell proliferation and transformation. Biochem. Biophys. Acta, 1072: 129-157.

15. Boyle, W.J., T. Smeal, L.H.K. Defize, P. Angel, J.R. Woodgett, M. Karin and T. Hunter, 1991. Activation of protein kinase $\mathrm{C}$ decreases phosphorylation of c-Jun at sites that negatively regulate its DNA binding activity. Cell, 64: 573-584.

16. Brown, P.H., T.K. Chen and M. Birrer, 1994 Mechanism of action of a dominant-negative mutant of c-Jun. Oncogene, 9: 791-799.

17. Fanjul, A.N., H. Bouterfa, M. Dawson and M. Pfahl, 1996. Potential role for retinoic acid receptorg in the inhibition of breast cancer cells by selective retinoids and interferons. Cancer Res., 56: 1571-1577. 
18. Yang, L.-M., H.-T. Kim, D. Munoz-Medellin, P. Reddy and P.H. Brown, 1997. Induction of retinoid resistance in breast cancer cells by overexpression of cJun. Cancer Res., 57: 4652-4661.

19. Giannini, G., M.I. Dawson, X-k. Zhang and C.J. Thiele, 1997 Activation of three distinct RXR/RAR heterodimers induces growth arrest and differentiation of neuroblastoma cells. J. Biol. Chem., 272: 26693-26701.

20. Benbrook, D.M., S. Lu, C. Flanagan, J. Shen-Gunther, L.H. Angros and S.A. Lightfoot, 1997. Biological assay for activity and molecular mechanism of retinoids in cervical tumor cells. Gyn. Oncol., 66: 114-121.

21. Zacheis, D., A. Dhar, S. Lu, M.M. Madler, J. Klucik, C.W. Brown, S. Liu, F. Clement, S. Subramanian, G.M. Weerasekare, K.D. Berlin, M. Gold, J.R. Houck, K.R. Fountain and D.M. Benbrook, 1999. Heteroarotinoids inhibit the growth of head and neck cancer cell lines in vitro and in vivo through both RAR and RXR retinoic acid receptors. J. Med. Chem., 42: 4434-4445.
22. Dhar, A., S. Liu, J. Klucik, K.D. Berlin, M.M. Madler, S. Lu, P.J. Birckbichler, R.T. Ivey, D. Zacheis, C.W. Brown, E.C. Nelson and D.M. Benbrook, 1999. Synthesis and structure-activity relationships of nitrogen heteroarotinoids. J. Med. Chem, 42: 3602-3614.

23. Dignam, J.D., R.M. Lebovitz and R.G. Roeder, 1983 Accurate transcription initiation by RNA polymerase II in a soluble extract from isolated mammalian nuclei. Nucl. Acids Res., 11: 1475-1489.

24. Merlin, J.-L., 1994. Concepts of synergism and antagonism. Anticancer Res., 14: 2315-2320.

25. Eferl, R. and E.F. Wagner, 2003. AP-1: a double-edged sword in tumorigenesis. Nature Reviews Cancer, 3: 85968. 